\title{
Jump Mechanism of Electric Charge Transfer in Gallium Arsenide Exposed to Polyenergy Implantation with $\mathrm{H}^{+}$Ions
}

\author{
P. Żukowski ${ }^{a}$, P. WęGiereK ${ }^{a, *}$, P. Billewicz ${ }^{a}$, T.N. Koltunowicz ${ }^{a}$ \\ AND F. KOMAROV ${ }^{b}$ \\ ${ }^{a}$ Lublin University of Technology, Nadbystrzycka 38A, 20-618 Lublin, Poland \\ ${ }^{b}$ Belarussian State University, Minsk, Belarus
}

\begin{abstract}
The article presents the experimental results on electric conductivity investigations of gallium arsenide, exposed to polyenergy implantations with $\mathrm{H}^{+}$ions, depending on alternating current frequency $(50 \mathrm{~Hz} \div 5 \mathrm{MHz})$, testing temperature (liquid nitrogen temperature $\div 373 \mathrm{~K}$ ) and the temperature of 15 min isochronous annealing $(293 \div 663 \mathrm{~K})$. It has been found that the obtained dependences $\sigma\left(T_{p}, f\right)$ result from a jump mechanism of electric charge transfer between the radiation defects that form in the process of ion implantation. Correlations between annealing of various types of radiation defects and conductivity characteristics $\sigma\left(T_{p}, f\right)$ have also been discussed.
\end{abstract}

PACS: 61.72.uj, 61.72.Cc, 72.80.Ey

\section{Introduction}

Papers [1-3] discuss a possibility of applying GaAs polyenergy implantation with $\mathrm{H}^{+}$ions to produce vertical isolation of integrated circuits. Series of energy and fluence values have been selected for the implanted $\mathrm{H}^{+}$ ions in the process of computer simulation in order to obtain uniform distribution of radiation defects within the maximum depth of $c a .3 .5 \mu \mathrm{m}$ with the maximum ion energy usage at the level of $400 \mathrm{keV}$. Additionally, $15 \mathrm{~min}$ high-temperature sample annealing has ensured stabilization of electrical properties of vertical isolation layers. As a result of the performed research we have also collected a series of analyses concerning mechanisms of electric charge transfer in strongly defected GaAs. Among them, the most important are observations of the jumping recharging mechanism [4] and the elaborated model of that phenomenon, including both direct and alternating current [5]. Verification of the above-mentioned model has been discussed in [6].

\section{Analysis of the obtained results}

Figures 1 and 2 presents the Arrhenius graphs concerning a GaAs sample exposed to polyenergy implantation with $\mathrm{H}^{+}$ions. Characteristics have been plotted for a sample that has not been annealed and after 15 min of annealing within the $T_{\mathrm{a}}$ temperature range up to $663 \mathrm{~K}$. For the annealing temperatures of $T_{\mathrm{a}} \leq 473 \mathrm{~K}$,

\footnotetext{
* corresponding author; e-mail: p.wegierek@pollub.pl
}

no significant changes on the plots $\sigma\left(1000 / T_{p}\right)$ have been noticed (Fig. 1). Radiation defects that are typical for that $T_{\mathrm{a}}$ range can be characterized by the following values of activation energy, measured at the testing frequency of $1 \mathrm{kHz}$. For the inverted temperature ranging between $5<1000 / T_{p}<8$ activation energy value is $\Delta E_{1}^{L} \approx 0.17 \mathrm{eV}$. This radiation defect seems to be annealed at $T_{\mathrm{a}}=523 \mathrm{~K}$, which has caused conductivity increase by $c a .100$ times. For $3<1000 / T_{p}<4$ a radiation defect of $\Delta E_{2}^{L} \approx 0.85 \mathrm{eV}$ has occurred. Its annealing at $T_{\mathrm{a}}=523 \mathrm{~K}$ has caused conductivity decrease by about 8 times. For $1000 / T_{p}<3$ a radiation defect of $\Delta E_{3}^{L} \approx 1.1 \mathrm{eV}$ has been observed.

At the testing frequency of $1 \mathrm{MHz}$ (Fig. 2) within the temperature range of $5<1000 / T_{p}<8$ conductivity increase can be observed with $\Delta E_{1}^{H} \approx 0.19 \mathrm{eV}$. Then the conductivity value stabilizes, which is followed by its increase for the sample temperature of $1000 / T_{p}<2$ with the activation energy of $\Delta E_{2}^{H} \approx 0.95 \mathrm{eV}$. Temperature annealing at $T_{\mathrm{a}} \geq 523 \mathrm{~K}$ brings about a decay of this radiation defect and introduces a minimum value of $\sigma$ at $1000 / T_{p} \approx 3$. As long as the annealing temperature grows, that minimum increases up to the value of $\sigma_{\max } / \sigma_{\min } \approx 8$, at $T_{\mathrm{a}}=643 \mathrm{~K}$.

Figure 3 shows the plots of $\sigma\left(f, T_{p}\right)$ for a sample annealed at $T_{\mathrm{a}}=473 \mathrm{~K}$. As it can be seen, the $\sigma(f)$ dependence tends to weaken as long as the testing temperature increases. According to the formula proposed by Mott [7]:

$$
\sigma \sim f^{\alpha},
$$

the coefficient $\alpha$ is a weak function of frequency and for samples annealed at $T_{\mathrm{a}} \leq 473 \mathrm{~K}$ does not exceed the 


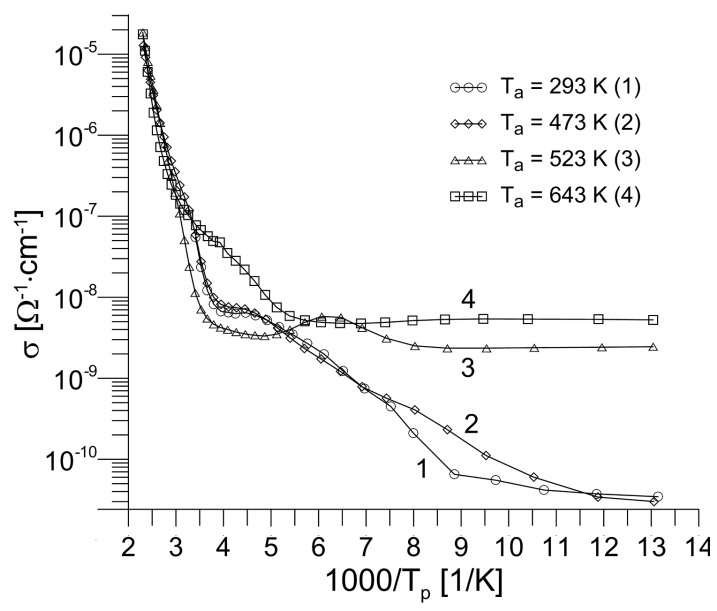

Fig. 1. Conductivity vs. inverted temperature for a testing frequency $1 \mathrm{kHz}$.

range from 0.7 to 1.0 (Fig. 5). This justifies the assumption that in the examined structure a jump mechanism of electric charge transfer has occurred.

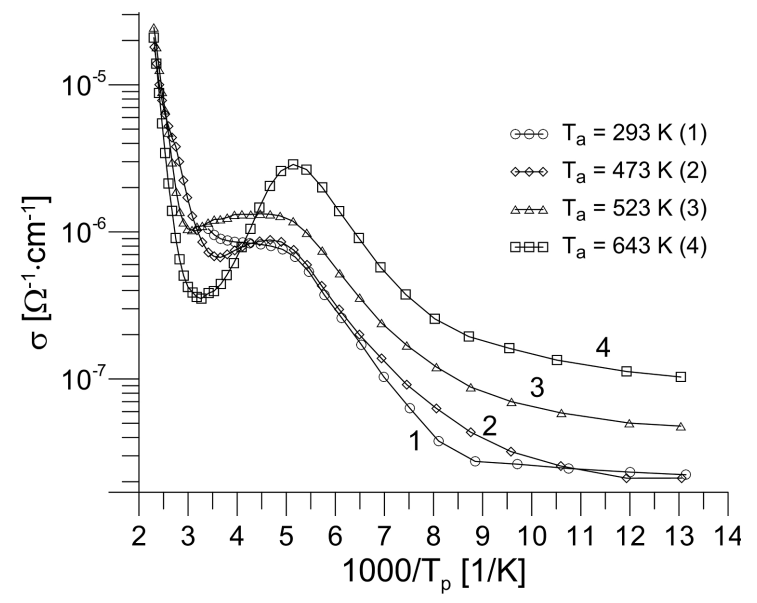

Fig. 2. Conductivity vs. inverted temperature for a testing frequency $1 \mathrm{MHz}$.

An increase of the value of $T_{\mathrm{a}}$ up to the level of $T_{\mathrm{a}} \geq 523 \mathrm{~K}$ leads to radical changes in the $\sigma\left(f, T_{p}\right)$ plots (Fig. 4). As the testing temperature rises, the $\sigma(f)$ dependence becomes stronger. It is possible to distinguish three characteristic parts on those plots. The first one comprising low frequency values $\left(f_{\mathrm{L}}\right)$ where it is difficult to observe any significant changes of $\sigma_{\mathrm{L}}(f)$. The second one corresponding to medium frequencies, where a rapid increase of $\sigma(f)$ is dominant and the third one that comprises higher frequencies, where $\sigma_{\mathrm{H}}(f)$ conductivity changes are also negligible. An analysis of the $\alpha(f)$ plot (Fig. 5) has shown that for the $\alpha$ coefficient the maximum value location within the frequency domain is strictly related to the shape of $\sigma(f)$ plots. Correlation between $\sigma$ and $\alpha$ has been explained using the model of jump conduction both for direct and alternating currents. In this model it has been assumed that once an electron has jumped from one neutral potential well to a second one, it stays there for a time $\tau$ and then jumps again into the third potential well with the probability $p$ in the direction determined by electric field. This phenomenon causes electrical conduction of direct current or low frequencies of alternating current. After the time $\tau$, the electron can also return to the first potential well with the probability $1-p$, which causes the high-frequency electrical conduction

$$
f \gg 1 /(2 \pi \tau) \text {. }
$$

In this case, the $\alpha(f)$ function has its maximum value at the frequency

$$
f_{\max } \approx 1 /(2 \pi \tau) \text {. }
$$

Probability of electron jumps is determined according to the formula [7]:

$$
P \sim \exp \left(-2 \alpha_{\mathrm{H}} R-\Delta E \tau / k T\right),
$$

where $\exp \left(-2 \alpha_{\mathrm{H}} R\right)$ is the rate of wave function decrease for an electron which is in the radiation defect potential well, $R$ - the average distance between radiation defects, $\Delta E_{\tau}$ - the jump activation energy, $\alpha_{\mathrm{H}}$ - the constant.

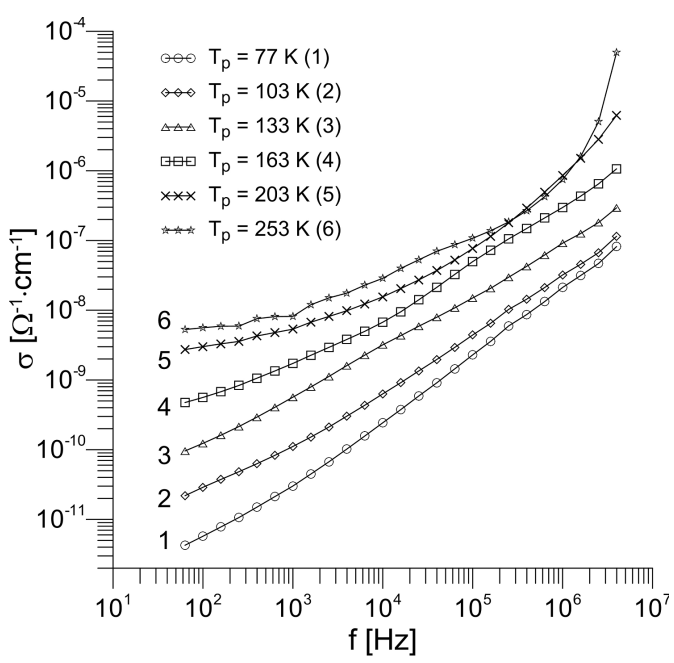

Fig. 3. Conductivity vs. testing frequency for a sample annealed at $T_{\mathrm{a}}=473 \mathrm{~K}$.

According to formula (4) time between two subsequent electron jumps is a function of temperature

$$
\tau=1 / P \sim \exp (\Delta E \tau / k T) .
$$

For the annealing temperatures of $T_{\mathrm{a}}>473 \mathrm{~K}$, the $\sigma(f)$ and $\alpha(f)$ dependences are in a good agreement with the characteristics predicted in the above-mentioned model. Thus, for low and high frequency values the correlation between conductivity and frequency is weak, while for medium frequencies a rapid growth of $\sigma(f)$ can be observed. The $\alpha(f)$ function has its local maximum that moves towards higher frequency values along with the 


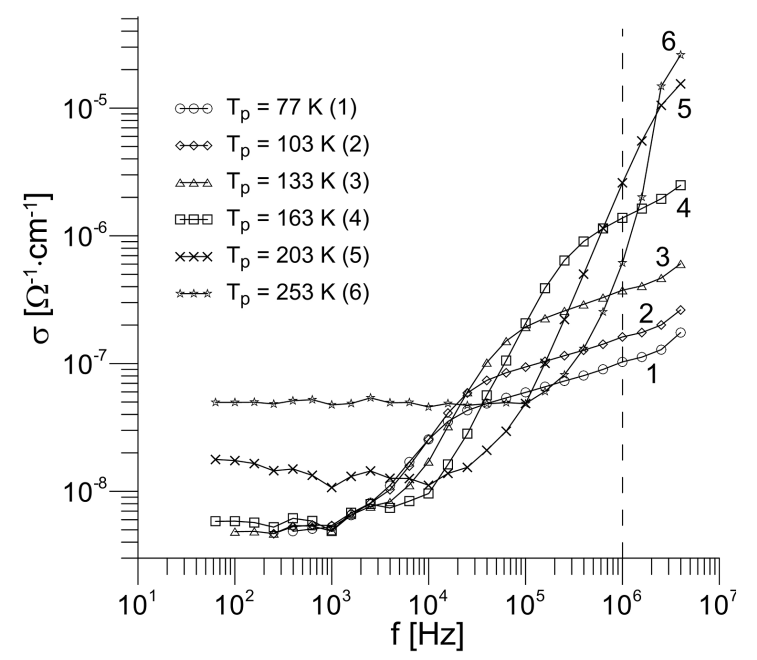

Fig. 4. Conductivity vs. testing frequency for a sample annealed at $T_{\mathrm{a}}=643 \mathrm{~K}$.

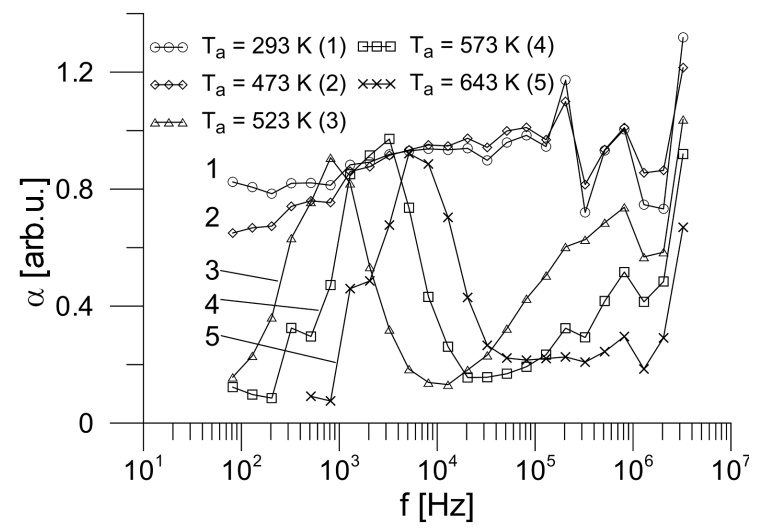

Fig. 5. Coefficient $\alpha$ vs. testing frequency, measured at $T_{p}=77 \mathrm{~K}$.

increasing temperature $T_{p}$, which means that the time $\tau$ decreases according to the formula (5) (Fig. 6).

An analysis of the $\sigma\left(f, T_{p}\right)$ characteristics shown in Fig. 4 makes it possible to explain the mechanism of a local minimum occurrence in the $\sigma\left(1000 / T_{p}\right)$ plot with the frequency of $1 \mathrm{MHz}$ (Fig. 2) by means of the jump conduction model, discussed above.

Rise of the testing temperature followed by simultaneous increase of the $\tau$ value is the reason why an area of the fastest $\sigma(f)$ increase rate moves towards higher frequencies. Up to the testing frequency value of $1 \mathrm{MHz}$ conductivity changes only insignificantly, the $\sigma_{\mathrm{H}}$ value increases with temperature (Fig. 2). As long as $T_{p}$ rises, the area of fast conductivity increase moves towards frequencies higher than $1 \mathrm{MHz}$. As a result, the conductivity value rapidly decreases at $1 \mathrm{MHz}$ (Fig. 4, plots 4, 5, 6).

In order to explain the absence of clear maximum values, like those that can be observed for $T_{\mathrm{a}} \leq 473 \mathrm{~K}$, it is necessary to analyze the $\alpha\left(f, T_{p}=77 \mathrm{~K}\right)$ characteristics presented in Fig. 5 for various annealing temperatures.

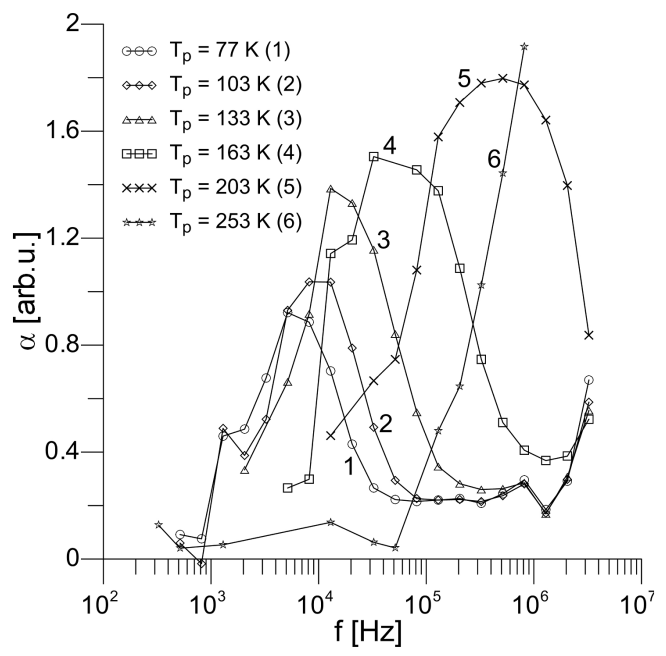

Fig. 6. Coefficient $\alpha$ vs. testing frequency for a sample annealed at $T_{\mathrm{a}}=643 \mathrm{~K}$.

As can be seen in the plot $\sigma(f)$, with the annealing at $T_{\mathrm{a}}=523 \mathrm{~K}$ being completed two local maximum values show up at the frequency values of $f_{1} \approx 8 \times 10^{2} \mathrm{~Hz}$ and $f_{2} \approx 8 \times 10^{5} \mathrm{~Hz}$. This means that in GaAs annealed at that temperature at least two different types of radiation defects are present. By analyzing the plots shown in Fig. 5, it is easy to observe that annealing at $T_{\mathrm{a}}=523 \mathrm{~K}$ is responsible for eliminating those defects that have their maximum value of $\alpha$ coefficient located in the vicinity of $f_{X} \approx 2 \times 10^{4} \mathrm{~Hz}$. Superposition of characteristics plotted for a couple of radiation defects with different time $\tau$ rates makes it possible to obtain dependences presented in Fig. 5, plots 1, 2. According to Fig. 1, when the annealing of an examined sample at $T_{\mathrm{a}}=523 \mathrm{~K}$ has been performed radiation defects of activation energy $\Delta E_{1}^{L} \approx 0.17 \mathrm{eV}$ and $\Delta E_{2}^{L} \approx 0.85 \mathrm{eV}$ tend to vanish. In our opinion, the absence of those defects in a sample annealed at $T_{\mathrm{a}}=523 \mathrm{~K}$ is responsible for introducing a local minimum in the characteristics that correspond to the $T_{\mathrm{a}} \geq 523 \mathrm{~K}$ temperature level, shown in Fig. 5.

\section{Conclusions}

In the presented article we have discussed an effect of polyenergy implantation with $\mathrm{H}^{+}$ions and $15 \mathrm{~min}$ high-temperature annealing on the temperature and frequency dependences of conductivity and $\alpha$ coefficient of the formula (1).

It has been established that dependences of conductivity on the inverted temperature $\left(1000 / T_{p}\right)$ do not change significantly as long as the sample annealing temperatures are maintained below the level of $T_{\mathrm{a}}=473 \mathrm{~K}$.

When the annealing at $T_{\mathrm{a}} \geq 523 \mathrm{~K}$ is performed, changes in the $\sigma(f)$ plots can be seen. Some areas of rapid conductivity growth separated from each other by the segments of much slower rate of $\sigma$ changes have been 
distinguished. For the $\alpha(f)$ dependences, it has been found that the occurrence of local maxima results from the mechanism of jump conduction that is specific for two types of radiation defects. According to the performed analyses, those defects have different values of the time $\tau$. At higher values of $T_{\mathrm{a}}$ a gap between $\alpha$ maximum points can be seen, unlike the case of lower $T_{\mathrm{a}}$ values, when such gap cannot be observed. In our opinion, this is related to the annealing at $T_{\mathrm{a}}=523 \mathrm{~K}$ of two different types of defects of activation energy values $\Delta E_{1}^{L} \approx 0.17 \mathrm{eV}$ and $\Delta E_{2}^{L} \approx 0.85 \mathrm{eV}$.

\section{References}

[1] F.F. Komarov, A.S. Kamyshan, A.M. Mironov, A.E. Zagutin, I.S. Martynow, Vacuum 63, 571 (2001).

[2] M. Kowalski, J. Partyka, P. Węgierek, P. Żukowski, F.F. Komarov, A.V. Jurchenko, D. Freik, Vacuum 78, 311 (2005).
[3] P. Żukowski, M. Kowalski, J. Partyka, P. Węgierek, T. Kołtunowicz, F.F. Komarov, A.S. Kamyshan, A.M. Mironov, A.V. Jurchenko, in: X Int. Conf. Physics and Technology of Thin Films, Iwano-Frankowsk, 2005, p. 181.

[4] P. Węgierek, P. Żukowski, T. Kołtunowicz, J. Partyka, F.F. Komarov, A.V. Jurchenko, in: The 6th Int. Conf. "Interaction of Radiation with Solids", Minsk 2005, p. 92.

[5] P. Żukowski, T. Kołtunowicz, J. Partyka, P. Wegierek, F.F. Komarov, A.M. Mironov, N. Butkievith, D. Freik, Vacuum 81, 1137 (2007).

[6] P. Żukowski, T. Kołtunowicz, J. Partyka, P. Wegierek, M. Kolasik, A.V. Larkin, J.A. Fedotova, A.K. Fedotov, F.F. Komarov, L.A. Vlasukova, Przeglad Elektrotechniczny 84, 247 (2008) (in Polish).

[7] N.F. Mott, E.A. Davis, Electronic Process in Non-Crystalline Materials, Clarendon Press, Oxford 1979. 